\title{
Auszug ans einem Schreiben des Hern Dr. E. Hartwig, Observator der Sternwarte in Dorpat, an den Herausgeber.
}

Als ich aın 19. März d. J. in Strassburg den Veränderlichen V Cygni beobachtete, fiel $\mathrm{mir}$ in seiner Umgebung ein Sternchen auf, welches ich bisher zu den Zeiten des Minimums von $\mathrm{V}$ nicht beachtet hatte. Da dasselbe auch heller als benachbarte, in der Durchmusterung vorkommende Sterne war, bestimmte ich sofort am i 8 zölligen Refractor, an welchem ich an jenem Abend mit anderen Beobachtungen beschäftigt war, seine und des Sternes DM. $+47^{\circ} 3167$ Position im Vergleich zu DM. $+47^{\circ} 3^{1} 5^{8}$ (AOe. 20888), welche Vergleichung für I 884.0 ergiebt:

$$
\begin{aligned}
& x \quad 20^{\mathrm{h}} 37^{\mathrm{m}} \text { I } 1888 \quad 47^{\circ} 49^{\prime} 7^{\prime \prime} \text { 。 } \\
& \begin{array}{lllllll}
47^{\circ} 3167 & 20 & 37 & 24.08 & 47 & 53 & 5.7
\end{array}
\end{aligned}
$$

Beim Nachsehen meiner Notizen uber V Cygni fand ich später, dass Birmingham in Nr. $2377 \mathrm{Bd}$. 100 der A. N. bei der Mittheilung seiner Auffindung des tiefrothen Sternes $V$ eines weissen, auch nicht in der DM. vorkommenden Sternes 9.1 $10^{\mathrm{m}}$ Erwähnung thut, welcher dem Stern DM. $+47^{\circ} 3_{167}$ auf Parallel etwa I 2 Secunden vorangeht. Auch Lindemann hat diesen letzteren Stern beobachtet und führt ihn unter der Birmingham'schen Position an (Bull. der Petersb. Akad. 'Tom. $29 \mathrm{Nr}, 2$ ). Weder am 19. März, noch kürzlich am 26. Juni in Dorpat, wo allerdings die helle Dämmerung um Mitternacht sehr störend wirkte, habe ich diesen weissen Stern an dem angegebenen Orte gesehen, vorausgesetzt, dass derselbe nicht identisch mit DM. $+47^{\circ} 3167$ oder erheblich schwächer ist. Dem von mir als DM. $+47^{\circ} 3167$ angesehenen Stern folgt etwa $15^{\circ}$ um etwa I' südlicher ein Sternchen IO. I I $^{\mathrm{m}}$. Gleichwohl ist der Stern $x$ nicht identisch mit Birmingham's white star, und da Birmingham seiner nicht erwähnt und meine Helligkeitsschätzung an 26. Juni von der am 19. März nicht unerheblich verschieden ist, so verdient dieser Stern einige Aufmerksamkeit. Auf meine briefliche Anfrage bezüglich der Position des white star hatte Herr Lindemann die Gefälligkeit, mir zu schreiben, dass die Birmingham'sche Angabe, wenn auch nicht ganz, doch nahezu zutreffe und dass in seinen photometrischen Messungen der verdächtige Stern $x$ zweimal vorkomme: 1882 Sept. $6=$ $9 \cdot 4$ und 188.3 Aug. $3 \mathrm{I}=9^{\mathrm{m}} \cdot 4$.

Bezilglich der Periode von V Cygni, welche Lindemann aus dem ihm zugänglich gewesenen Material vorläufig zu I 2 Monaten fand, bemerke ich, dass meine Beobachtungen, welche allerdings gerade zu den Hauptepochen wegen meiner zweimonatlichen Reise nach Russland und Schweden Anfangs 1882 und der Theilnahme an den Venusexpeditionen, ferner in Folge der schlechten Witterung des vergangenen Winters, grosse Lücken aufweisen, diese Periode nicht unter I 3 Monaten anzunehmen erlauben, so dass in diesem Jahre erst im September oder October das Maximum erwartet werden darf.

\section{Ueber die Sterne in der Ungegend ron $V$ Cygni.}

Im Anschlusse an vorstehendes Schreiben des Herrn Dr. Hartwig erlaube ich mir, Folgendes zu bemerken:

In A. N. 2377 sagt Herr J. Birmingham nicht, dass V Cygni, der "white star und DM. $+47^{\circ} 3$ I 67 zugleich am Himmel stehen, sondern lässt ausdruicklich die Frage offen, ob überhaupt und event. welcher der beiden erstgenannten Sterne mit dem Durchmusterungssterne identisch sei. Da nun seine Positionsbestimmung ron V Cygni, wie aus den von mir a. a. O. mitgetheilten Originalen der DM. und einer Positionsbestimmung von Prof. Krueger hervorgeht, $u m+10^{\text {s }}$ $z \mathrm{u}$ corrigiren ist, so ist als sehr wahrscheinlich anzunehmen, dass dieselbe Correction an die angegebene Position des white star anzubringen ist und dass demzufolge dieser mit DM. $+47^{\circ} 3167$ zusammenfällt.

Fraglich wird diese Correctur aber wieder dadurch, dass Herr Lindemann in seinem Aufsatze des Vorhandenseins des »white star neben dem Durchmusterungsstern ausdriucklich Erwähnung thut. Herr Dr. Lamp hat deshalb die Güte gehabt, auf meine Bitte die betreffende Stelle am Himmel nachzusehen und die etwa in Betracht kommenden Sterne durch mikrometrischen Anschluss an DM. $+47^{\circ} 3^{1} 5^{8}$ $=$ AOe. 20888 festzulegen.

Kiel 1884 Juli 26 .
Die von ihm gefundenen Positionen, auf 1855.0 reducirt,

\begin{tabular}{|c|c|c|c|}
\hline & $9 \cdot 2$ & $20^{h} 3^{6^{\prime \prime \prime}} 15^{5 \cdot 7}$ & $+47^{\circ} 43^{\prime}$ \\
\hline & $9 \cdot 5$ & 28.0 & 47 \\
\hline & 10 & 44.6 & 454 \\
\hline
\end{tabular}
sind folgende:

Alle drei Sterne kommen auch bei Dr. Hartwig vor, der erste als Stern $x$, der zweite $=$ DM. $+47^{\circ} 3$ I 67 .

Der » white star « ist hier, ebenso wie in Dorpat, nicht gesehen worden.

Es scheint mir hiernach im Zusammenhange mit dem Schreiben ron Dr. Hartwig die Möglichkeit vorzuliegen, dass der white star in der Birmingham'schen Position iberhaupt nicht existirt hat, und dass der von Herrn Lindemann als solcher bezeichnete Stern DM. $+47^{\circ} 3{ }^{\circ} 67$ ist, dagegen der von ihm mit DM. $+47^{\circ} 3 \times 67$ identificirte Stern in der DM. nicht vorkommt und mit dem von Dr. Lamp bestimmten Sterne $c$ zusammenfällt.

Herr Lindemann wird sicher in der Lage sein, über die Richtigkeit dieser Annahme genauere Auskunft ertheilen zu können.

\section{H. Kreuts.}

